\title{
15 Die kategoriale Minimalform von „Realität überhaupt“: Setzende, äußere, bestimmende Reflexion
}

„Diese reine absolute Reflexion, welche die Bewegung von Nichts zu Nichts ist, bestimmt sich selbst weiter" (GW 11, S. 250) - so lapidar kennzeichnet Hegel nun die Notwendigkeit, der drohenden Abstraktheit und Innerlichkeit des Wesens als reiner Reflexion entgegenzutreten, indem die Erinnerung an die seinslogische Verantwortlichkeit durch das Wesen als Reflexion selbst wiederaufgenommen wird: und zwar dadurch, dass sich diese reine Reflexion selbst weiterbestimmt, also aus den eigenen logischen Funktionen und Bedingungen heraus Leerstellen benennt, die durch weitere Entfaltung der Struktur reflexiver Negation erst noch gefüllt werden müssen, um den Ansprüchen des Wesens an die „Wahrheit“, als welche es gleich zu Beginn gesetzt worden ist, Genüge zu tun. Dies kann nur gelingen, indem die Art des Übergangs zwischen reflektierter Unmittelbarkeit und reflexiver Negativität, die logischen Konsequenzen, die sich aus seiner Verfasstheit ergeben sowie deren noch unentfaltete Bedingungen (also das gesamte Geschehen des Scheinens des Wesens in sich selbst) nochmals näher betrachtet werden. Ziel ist es, die drohende mechanische Geschlossenheit, Innerlichkeit und Abstraktheit dieses letztbegründenden Vollzuges reinen „Bestimmtseins überhaupt“ aufzubrechen und damit die Anschlussstellen sowie Argumentationsperspektiven zu öffnen, die in den Reflexionsformen verborgen liegen. Man könnte auch sagen, die drei Reflexionsformen explizieren kritisch den Bedeutungsgehalt der reinen absoluten Reflexion, indem sie gemäß der hegelschen Einheit von Darstellung und Kritik die nähere Thematisierung derselben als kritische Überschreitung und Entfaltung ins Werk setzen: Die Reflexionsformen sind verschieden perspektivierte wie zugleich progressiv zusammenhängende Metabestimmungen der reinen Reflexion. Mit den Reflexionsformen gibt sich die reine absolute Reflexion (1.) selbst ein explizites Wissen von ihrer Arbeitsweise und den operativen Funktionen, aus denen danach die Kategorien der Wesenslogik hervorgehen sollen, und sie gibt sich (2.) die Handlungsprozeduren, mit denen sie die Entfaltung der wesenslogischen Bestimmungen - den Bedingungen des Wesens gemäß - vollziehen kann. Rezeptionsgeschichtlich gesehen reformuliert Hegel mit den Reflexionsarten die drei Grundsätze Fichtes aus der Wissenschaftslehre ${ }^{114}$. Bereits darin liegt der Hinweis, die ursprünglich mit diesen Grundsätzen erhobenen Geltungsansprüche bei Fichte auf den Entwurf des Grundschemas kategorialen

114 Vgl. Iber: Metaphysik absoluter Relationalität, S. 142.

Ә OpenAccess. (c) 2021 Claudia Wirsing, publiziert von De Gruyter. (cc) BY Dieses Werk ist lizenziert unter einer Creative Commons Namensnennung 4.0 International Lizenz. 
„Realseins überhaupt“ (vgl. Kap. 3 im Hauptteil I) auch zumindest als thematischen Hintergrund der hegelschen Begriffsbildung zu verstehen.

\section{Setzende Reflexion}

Die Stufe der „setzenden Reflexion“ expliziert die reine absolute Reflexion unter der bereits beschriebenen Problemstellung, alles Andersseins verschwinden zu lassen. Zugleich entdeckt sie aber gerade in deren Mechanismen das Potenzial der Überschreitung dieser Gefahr. Ich will mich im Folgenden auf eben jene zentralen Überschreitungsperspektiven konzentrieren.

Die reine absolute Reflexion, so beginnt Hegel den Abschnitt, „besteht also darin sie selbst und nicht sie selbst und zwar in Einer Einheit zu seyn“ (GW 11, S. 250), wobei „sie selbst“ hier die sprachliche Codierung der wesenslogischen Unmittelbarkeit ist, indem in der Verstärkung der Apposition des „selbst“ die Beziehung der „einfache[n] Gleichheit mit sich“ gemeint ist. Die setzende Reflexion geht also von der Bestimmung aus, dass die ,sich auf sich beziehende Negativität“ (GW 11, S. 250) als Anfang, Kern und Gesamtstruktur der reinen absoluten Reflexion auch im „Negiren ihrer selbst“ (GW 11, S. 250), also darin, „nicht sie selbst“ zu sein, doch ganz bei sich als Negativität ist.

Die sich auf sich beziehende Negativität ist sowohl die einfache Unmittelbarkeit, die sie an sich hat - als Sichselbstgleichheit der reflexiven Negation -, als auch das durch ihre Selbstnegation entstehende (d.h. gesetzte) Andere zu ihr, die reflektierte Unmittelbarkeit als Produkt der Aufhebung der Negation durch sich, die doch als Unmittelbarkeit zugleich nichts anderes als diese Negativität ist. Der Aspekt des „Gesetzseyn[s]“ (GW 11, S. 251) dieser Unmittelbarkeit als das Andere zur reflexiven Negativität betont mithin den Umstand, dass diese Unmittelbarkeit auf unmittelbare Weise an ihr selbst reflektiert, d. h. aufgehoben ist, weil sie eben aufgrund ihres Vermitteltseins durch Negativität immer schon nichts anderes als die Negativität ist. In dieser Unmittelbarkeit verlässt sich die Negativität nicht (nur), sondern sie kehrt (auch) in sich zurück, ist also ,so sehr a ufgehobene Negativität als sie Negativität ist“ (GW 11, S. 250). Als gesetzte Unmittelbarkeit ist diese „nur als Rückkehr des Negativen in sich“ (GW 11, S. 251): nämlich gänzlich Vermittlungsprodukt selbstbezüglicher Negativität und damit bloßer Schein von Unmittelbarkeit, verstanden als Anderes zur Negativität.

Im Aspekt des „Gesetztseins“ der Unmittelbarkeit innerhalb der reinen absoluten Reflexion beschwört Hegel in nochmals intensivierter Form die Gefahr eines logischen Konstruktivismus reinen Denkens als Selbstbeschreibung wesentlichen Bestimmtseins: nämlich alle an sich selbst als ungedacht bestimmten Gegenständlichkeiten einzig in der Weise des Effekts reiner begrifflicher Ver- 
mittlung zu fassen, als deren Produkt und damit, ihrem Sein nach, letztlich bloß als „Gedankendinge“. Das gleitende, unmittelbare Ineinanderübergehen von Negation und Unmittelbarkeit, das sich unter der Regierung der reinen selbstbezüglichen Negativität vollzieht, lässt die logische Eigenzeit der Andersheit ${ }^{115}$ des Unmittelbaren, die seine Selbstständigkeit betont, verschwinden. In der von Hegel deutlich betonten „Einheit“ der setzenden Reflexion in ihrer Ausgangsformation hat der bestehende Unterschied von Negativität und Unmittelbarkeit keinen $\mathrm{Be}$ stand, weil er stets wieder in die Einheit der sich negierenden Negativität zurückfällt. Somit ist der erste Befund, der über die reine absolute Reflexion in Gestalt des Aspekts ihres Gesetztseins ausgesprochen wird, hochgradig unbefriedigend. Denn eine sinnvolle kategoriale Matrix minimalen „Realseins überhaupt“ muss die Beständigkeit des Anderen als Herausforderung und Bedingung ihres Begriffs in eine logische Eigenzeit des Auseinander und Gegeneinander der „Bestimmungselemente überhaupt“ übersetzen können: d. h. sie muss räumliche Verhältnisse in der zeitlichen Übergängigkeit von Unmittelbarkeit und absoluter Negativität verorten. ${ }^{116}$

Eben hier setzt nun der nächste hochbedeutsame Gedankenschritt innerhalb der „setzenden Reflexion“ an:

Die Reflexion ist also die Bewegung, die, indem sie die Rückkehr ist, erst darin das ist, das anfängt oder das zurückkehrt.

Sie ist Setzen, insofern sie die Unmittelbarkeit als ein Rückkehren ist; es ist nemlich nicht ein anderes vorhanden, weder ein solches, aus dem sie, noch in das sie zurückkehrte; sie ist also nur als Rückkehren oder als das Negative ihrer selbst. Aber ferner ist diese Unmittelbarkeit die aufgehobene Negation und die aufgehobene Rückkehr in sich. Die Reflexion ist als Aufheben des Negativen Aufheben i hres A nd ern, der Unmittelbarkeit. Indem sie also die Unmittelbarkeit als ein Rückkehren, Zusammengehen des Negativen mit sich selbst ist, so ist sie eben so Negation des Negativen als des Negativen. So ist sie Vor a u ssetzen. (GW 11, S. 251)

Hegel geht jetzt dazu über, von den Produkten der reinen absoluten Reflexion auf deren Tätigkeitsweisen zurückzugehen, d.h. vom „Gesetztsein“ auf das „Setzen“ als reinen Akt zurückzublicken. Ziel ist es dabei, durch die weitere und noch grundlegendere Entfaltung der Logik reflektierter Unmittelbarkeit im Wesen, also durch eine noch genauere und feinere Kritik der Arbeitsweise ihres Zustandekommens inner-

115 „Logische Eigenzeit“ meint hier, dass die gesetzte Unmittelbarkeit auf unmittelbare Weise sofort in ihrem Entstehen zugleich als Negativität, d.h. als dasselbe wie ihr Grund, erkannt wird und deshalb zu keinem logischen Zeitpunkt von diesem wirklich unterschieden ist.

116 Zeitlich schmilzt alles zum selben zusammen; räumlich tritt es in Gegenstände, als gegeneinander Stehendes, auseinander. 
halb der selbstbezüglichen Negativität, die bloße Immanenz und Geschlossenheit der setzenden Reflexion aufzubrechen. Schließlich muss stets mitbedacht werden, dass Hegel in jedem Fall - auch wenn die Frage nach minimaler kategorialer „Realität überhaupt“ ,lediglich“ eine Interpretationsperspektive der vorliegenden Arbeit und nicht exoterisches Programm ist - in der Wesenslogik das explizit formulierte Ziel verfolgt, die Gefahr einer bloßen Immanenzstruktur des Wesens, wie sie zu Anfang der Wesenslogik als Hypothek der klassischen Metaphysik entworfen wird, gänzlich hinter sich zu lassen. Auch in der „Verstandesphilosophie“ des Wesens sollen bereits die Richtungen auf eben jene echte „Wahrheit“ aufgespürt werden, als die das Wesen zu Beginn angesprochen wird. Hegel zeigt nun, dass die Bestimmung der reinen Tätigkeit selbstbezüglicher Negativität nur als „Setzen“ logisch unvollständig ist: dass also Setzen, gedacht in seiner kategorialen Form als reine Bestimmungshandlung des „Begrifflichen überhaupt“, in seiner Geltung nicht als selbstgenügsam zu fassen ist. Hegel macht klar, dass gerade aufgrund der Art der schrankenlosen Übergängigkeit von selbstbezüglicher Negativität und reflektierter Unmittelbarkeit ineinander (nämlich Übergängigkeit als negatives Verhältnis zu sein) die produzierende Negation eben der Negativität auch untersteht, durch welche sie ihr Produkt bestimmt und die sich in der gesamten Relation als ihr Selbstverhältnis ausbildet. Pointiert gesagt: Die sich negierende Negation entkommt sich selbst nicht, weil sie das ganze Verhältnis bestimmt; deshalb verschwindet sie ebenso, wie sie das seinslogische Unmittelbare verschwinden lässt. Deshalb ist die Unmittelbarkeit nicht nur „Zusammengehen des Negativen mit sich selbst“, sondern auch „Negation des Negativen als des Negativen“ (GW 11, S. 251): D. h. (1.) dass in der gesetzten Unmittelbarkeit nicht nur die selbstbezügliche Negativität im Verlassen ihrer selbst zu sich zurückkehrt und sich so zuallererst gewinnt, weil sich die gesetzte Unmittelbarkeit als reflektierte, immer schon verneinte und nichts anderes als Negativität seiende erweist. (2.) Im Setzen der Unmittelbarkeit verliert sich die selbstbezügliche Negation auch, d.h. in ihr verschwindet zugleich auch umgekehrt das „Negative überhaupt“, weil die Unmittelbarkeit kraft ihres Gehalts den Prozess ihrer Erzeugung zugleich auch zum Verschwinden bringt. ${ }^{117}$ Buchstabiert man den Setzungsprozess selbstbezüglicher Negativität vollständig aus, so wird das logische Moment sichtbar, durch das er sich selbst unterbricht und seiner Immanenz entweicht - durch das er logisch als angewiesen auf eine Anerkennung von Andersheit erscheint, die weder wie im bloßen Sein ein ganz Äußeres zu ihm ist, noch gänzlich

117 Nach Anton Friedrich Koch hebt Hegel durch die selbstbezügliche Negation die Widersprüchlichkeit unseres Denkens in der prozessualen Entwicklung des logischen Raums auf (Koch: Die Evolution des logischen Raums, S. 308). Der selbstbezüglichen Negation kommt somit eine therapeutische Funktion zu, indem sie das Widerspruchsprinzip zur „Selbstkorrektur des Denkens“ (Koch: Die Evolution des logischen Raums, S. 133) erhebt. 
(im Sinne eines reinen Immanenzbegriffs des Wesens) der Verfügung des Setzens untersteht. Die Tätigkeit heißt dort, wo sie diese Aufhebung als Unterbrechung ihrer selbst in Szene setzt, eine voraussetzende und die Unmittelbarkeit eine vorausgesetzte. Hegel gelingt es an dieser Stelle erneut, einen scheinbar selbstverständlich gewordenen Begriff der Alltagssprache, der in ihr sogar weitaus gebräuchlicher als der des „Setzens“ ist, philosophisch aufzuladen und zugleich auf komplexe Weise neu zu entfalten. Demnach gilt: „Als Voraussetzen läßt die Reflexion der Unmittelbarkeit eine nicht nur phantasmagorische Selbständigkeit zuwachsen. Mithin ist die Unmittelbarkeit nicht mehr bloße Bestimmtheit oder sich reflektierend, sondern unabhängig und in stärkerem Sinne ein Anderes der Reflexion als das ursprünglich Gesetzte.“118

Das Voraussetzen als Aspekt, unter dem der unendliche Vollzug des negativen Setzens sich selbst aussetzt (d.h. unterbricht und zum Verschwinden bringt), ist aber auch deshalb der einzig adäquate Fortgang der Beschreibung der reinen absoluten Reflexion, weil in ihm die Wesenslogik den Ergebnissen ihres bisherigen Gangs wieder entsprechend wird: Setzen, Voraussetzen und Aussetzen bilden die Bedingung dafür, auf der Stufe der reinen absoluten Reflexion die logische Verantwortlichkeit gegenüber dem Schein wieder anzuerkennen. Unmittelbarkeit ist durch das Setzen selbstbezüglicher Negativität auch als vor ihr liegend bestimmt, d. h. als ihr auch vorausgehend und auch von ihr unabhängig vorliegend. Das aber ist kein Widerspruch, der stets nur wieder auf das Gleichsein der Unmittelbarkeit mit dem „durch“ des Setzens und Gesetztseins und damit auf einen Konstruktivismus des Setzens zusammenschmilzt, der in allen von ihm abhängigen Prozessen nichts anderes als er selbst ist. Die Reflexionsformen sind eben der Ort in der Logik, wo diese schwierige Grenzfigur, die zugleich nach dem hier gemachten Vorschlag als Begriff minimaler kategorialer „Realität überhaupt“ zu denken ist, entfaltet und begründet wird: wie sich die reflektierte Unmittelbarkeit, dieser „Schein“ ihrer selbst, als von der Reflexion bestimmte, d.h. als unbestimmt bestimmte $^{119}$, von diesem „Bestimmtwerden überhaupt“ aspektional freistellt, indem sie eine Unabhängigkeit gegen das sie setzende Bestimmen geltend macht,

118 Iber: Metaphysik absoluter Relationalität, S. 152.

119 Damit geht Hegel an dieser Stelle auch über seine Kritik des kantischen „Ding an sich“ hinaus, wie sie vor allem in der Enzyklopädie zu finden ist. Denn dort hatte er Kants ontologische Unterscheidung dadurch kritisiert, dass in ihr eigentlich nur die Bestimmung des Unbestimmten als leichteste, einfachste, aber auch leerste überhaupt gesetzt worden sei (vgl. GW 20, S. 80 f., § 44) und somit das Bestimmen die Unbestimmtheit regiert. In der Logik der Reflexionsformen hingegen versucht er dem begrifflichen Anspruch gerecht zu werden, dass das als unbestimmt Bestimmte in Aspekten seiner Unbestimmtheit nicht dem ursprünglichen Bestimmen weiter untersteht. 
welches nicht der initialen Aktionsform des Bestimmtwerdens bloß untersteht und doch zugleich kein eigentliches Außen gegen dieses einnimmt. Mit anderen Worten: Hegel denkt an diesem Punkt den Umstand, dass dem reflektierten Unmittelbaren eine Dimension der Geltung seines Gehalts zukommt (Unabhängigkeit vom Setzen), die nicht den Bedingungen seiner logischen Genese (Gesetztwerden) untersteht und doch diese nicht außer Kraft setzt. Somit umreißt der Abschnitt zur „setzenden Reflexion“ klar die Aufgabe: Aus dem Inneren der Logik reiner Reflexion und unter Beachtung der Norm seinslogischer Verantwortlichkeit muss die Gegenläufigkeit der Unmittelbarkeit in ihrer Bestimmtheit durch die Reflexion verstanden werden, um gegen die Bedingungen der Genese ihres Gesetztseins durch Reflexion einen Aspekt von Geltung an ihr aufzuspüren, die diesen Bedingungen nicht untersteht und nicht nur deren Effekt ist, ohne ihnen ganz äuBerlich zu sein.

Weder ist deshalb Manfred Frank zuzustimmen, der in Überspitzung dieser Gegenläufigkeit im Voraussetzen bei Hegel die Rückkehr eines völlig negationsfreien ontologischen Fundamentes sieht, mit dem sich schließlich die Abhängigkeitsrichtung gänzlich umkehrt und die Reflexion als Epiphänomen absolut vorgängiger, unmittelbarer und undifferenzierbarer Einheit eines schellingschen oder hölderlinschen „Seins“ entlarvt wird. ${ }^{120}$ Noch ist Christian Iber gegenteilig zuzustimmen, der diese Dimension von Unmittelbarkeit letztlich wieder nur als leeren Schein begreift, der als durch das Wesen selbst gesetzter am Ende der Betrachtung keine wirklich gleichberechtigte Geltung gegenüber dem Gesetztsein nach sich zieht und eben nur Funktionsweise der Selbstentfaltung des Setzens der Reflexion ist. ${ }^{121}$ Bereits in der „setzenden Reflexion“ markiert der Aspekt des Voraussetzens eben jene Grenze, auf der die logische Figur minimaler kategorialer Realität ihren Ort hat: Die Unmittelbarkeit ist demzufolge nicht bloßer Schein, sondern wiederhergestellter Schein im Sinne seinslogischer Verantwortlichkeit, und muss also in ihren Ansprüchen für die Beschreibung der „Reflexionsform überhaupt“ ernst genommen werden.

Möglich wird diese Denkfigur, weil durch das Voraussetzen eine zweite Richtung des Bedingtseins in die reine absolute Reflexion eingeführt wird, die gegenüber der ersten kein leerer Schein ist. Vielmehr gilt, dass diese zweite Richtung zwar als durch die erste Richtung entdeckte, aber nicht gänzlich kon-

120 Vgl. hierzu Manfred Frank: Der unendliche Mangel an Sein. Schellings Hegelkritik und die Anfänge der Marxschen Dialektik, 2. Aufl., München 1992, S. 46-50.

121 Iber: Metaphysik absoluter Relationalität, S. $156 \mathrm{f}$. 
stituierte verstanden werden darf. ${ }^{122}$ So erfolgt die Verdopplung des logischen Richtungssinns, der das Setzen als Genese der Unmittelbarkeit leitet und zwei geltungstheoretisch gleichwertige, wenn auch nicht gleichursprüngliche Bedingungsrichtungen sichtbar macht. Hierzu heißt es:

\begin{abstract}
Die Reflexion also fin d et ein Unmittelbares vo r, über das sie hinausgeht, und aus dem sie die Rückkehr ist. Aber diese Rückkehr ist erst das Voraussetzen des Vorgefundenen. Diß Vorgefundene wird nur darin, daß es verlass en wird; seine Unmittelbarkeit ist die aufgehobene Unmittelbarkeit. - Die aufgehobene Unmittelbarkeit umgekehrt ist die Rückkehr in sich, das A n k o m m en des Wesens bey sich, das einfache sich selbst gleiche Seyn. Damit ist dieses Ankommen bey sich das Aufheben seiner und die von sich selbst abstossende, voraussetzende Reflexion, und ihr Abstossen von sich ist das Ankommen bey sich selbst. (GW 11, S. 252)
\end{abstract}

Die „reflectirende Bewegung“ als „absoluter Gegenstoß in sich“, in welchem das „Hinausgehen über das Unmittelbare [...] vielmehr erst durch diß Hinausgehen“ (GW 11, S. 252) besteht, wodurch die „Bewegung [...] sich als Fortgehen unmittelbar in ihr selbst um[wendet] und [...] nur als Selbstbewegung “ (GW 11, S. 252) ist, muss als Explikation eines Zusammenhangs von gegenläufigen Bedingungsrichtungen und reinen logischen Geltungshandlungen verstanden werden. Dabei ist der „Schein des Anfangs“ (GW 11, S. 251), den die voraussetzende Reflexion erzeugt, im Sinne der seinslogischen Verantwortlichkeit des Scheins zu verstehen, um nicht in die Geschlossenheit des bloßen Setzens zurückzufallen. Indem die Reflexion die Unmittelbarkeit als vorausgesetzte bestimmt und somit deren Bedingung bildet, bestimmt sie sich zugleich als wiederum durch diese bedingt, d.h. als bedingt durch ein ihr Vorausliegendes, welches ihren Grund abgibt, weil es bestimmt ist als den Vermittlungs- und Bestimmungsvollzügen der Reflexion unverfügbar Vorgegebenes, von dem sie auszugehen hat. Wenn dieser zirkuläre Ablauf einsinnig im Verständnis seines Gehalts auf den initialen Setzungsakt der Reflexion zurückgeführt wird, geht zum einen seine echte Zirkularität verloren, zum anderen wird der in der Entwicklung des Scheins etablierte Anspruch seinslogischer Verantwortlichkeit wiederum fallen gelassen und so die von Hegel gerade kritisierte Immanenz des Wesens bestätigt.

Die Pointe dieser Entfaltung ist es vielmehr, dass die Reflexion im Aussetzen ihres Setzungsaktes durch das Voraussetzen ein Anderes (Unmittelbares) zur Reflexion als gegeben anerkennt, dessen Sein ihr darin unverfügbar ist, als sie von diesem auszugehen hat. Zugleich aber ist dieses Andere völlig für das Be-

122 Auch hier verhält es sich wie bei Fichte, wo nämlich das Nicht-Ich keineswegs vom Ich gesetzt, d.h. konstruiert, wird, sondern das Ich nur den Unterschied zum Nicht-Ich gesetzt und ihm damit eine Bestimmung gegeben hat (nämlich ein Nicht-Ich zu sein). 
stimmen der Reflexion offen, insofern es diesem vollständig entspricht und mit dem reflexionslogischen Bestimmen folglich demselben Raum von Bestimmtheit überhaupt („Selbstziehung“) zugehört. Sowohl die sich negierende Negation als auch die als vorausgesetzt gesetzte Unmittelbarkeit teilen denselben Grad von vollständiger Bestimmtheit im Sinne des Vermitteltseins durch begriffliche Strukturen. Die Reflexion der selbstbezüglichen Negativität als logische Grundform verstandesmäßigen „Bestimmens überhaupt“ gibt somit aus sich selbst, d.h. aus der Bedeutsamkeit ihres Vollzuges heraus, den Raum für die Geltung eines Unmittelbaren, ihrem Bestimmen in einer Hinsicht Vorausliegenden frei. Dieses Vorausliegende ist aber darin kein Anderes im Sinne des Seins mehr, als es auch in seinem Voraussein der vollständigen Bestimmbarkeit durch die Reflexion offen steht, insofern es nichts als Bestimmbarkeit und darin als immer schon notwendig bestimmt zu denken ist. Es hat dieses Bestimmtsein durch die Reflexion damit ursprünglich an sich, ohne gänzlich auf dieses zurückzugehen. Dass Unmittelbarkeit gesetzt ist durch den reinen Akt des Bestimmens, d.h. als reflektierte Unmittelbarkeit in diesem Setzen gründet, meint den Umstand, dass es als jeder reflexiven Bestimmung vorgängig an sich bereits bestimmt zu denken ist, damit es nachfolgend vom Bestimmen der „Reflexion überhaupt“ bestimmt werden kann. Beide Bestimmtheitsweisen sind vielmehr Konkretisierungsrichtungen eines übergreifenden logischen Raums von „Bestimmtheit überhaupt“, an dem sie gleichursprünglich partizipieren und diesen so erfüllen. Die Unmittelbarkeit darf nicht aus der Bestimmtheit des Wesens wie ein Äußerliches herausfallen (seinslogischer Realismus des Unmittelbaren), ohne zugleich ganz auf die einseitige Bestimmtheit des Setzens durch die Reflexion zusammenzuschrumpfen (wesenslogischer Konstruktivismus des Unmittelbaren). Die so etablierte Geltung des Vorausgesetzten gegen die Genese des Setzens wird demnach denkmöglich, wenn man Setzen und Vorausgesetztes bzw. Bestimmtsein des Vorausgesetzten durch das Setzen als gegen das Setzen, im Sinne einer inneren Differenzierung, versteht. Mit dieser realen Differenzierung umreißen beide Bestimmungsrichtungen zuallererst einen ihnen wiederum vorausliegenden logischen Raum absoluten Bestimmtseins, innerhalb dessen verschiedene Ebenen und Richtungen von Bestimmtsein und Bestimmtwerden als Bedingungsgefüge und zugleich Irreduzible widerspruchsfrei denkbar sind. Dieser logische Raum ist das Feld minimaler kategorialer „Realität überhaupt“, und er kann in kategorialer Analyse nur entdeckt werden, indem er sich als notwendig zu denkendes reines Beziehungsfeld aus den Bewegungen der reinen absoluten Reflexion ergibt. Eben diese Entwicklung wird in der ,äußeren Reflexion“ konsequent weitergeführt; sie zeigt sich schon im Übergang von der setzenden zur äußeren Reflexion, welcher noch im Abschnitt zur „setzenden Reflexion“ liegt. 


\section{2 Äußerliche oder reale Reflexion}

Die „äusserliche oder reale Reflexion“ (GW 11, S. 252) ist in der Forschung immer wieder und fast durchgängig in der Stigmatisierung als „schlechtes Verstandesdenken“ (um das es in der Wesenslogik auf einer ersten, exoterischen Ebene bekanntlich geht ${ }^{123}$ und welches in der äußeren Reflexion in der Tat am schärfsten und deutlichsten hervortritt) eindeutig negativ bewertet worden. Paradigmatisch hierfür ist v.a. Henrichs Aufsatz „Hegels Logik der Reflexion“ in seiner ersten Fassung (1971), in welchem er die äußere Reflexion zum einen als vollendeten Ausdruck eben jenes schlechten Verstandesdenkens und zum anderen innerhalb des Fortgangs der Reflexionsformen als bloßen Exkurs, der der Reflexion nichts Neues mehr hinzufüge und letztlich nur ihre generellen Abirrungen aufzeige, begreift. So vermerkt Henrich: „Wird also zum Gedankengang der ,setzenden Reflexion' nichts hinzugefügt, so kann auch die,äußere Reflexion' nicht mehr als eine Anmerkung sein. In ihr würde demnach dargelegt, was sich ergibt, wenn die Reflexion beim Voraussetzen ihre eigene setzende Tätigkeit vergessen macht.“124 Die äußere Reflexion gerinnt so zum Abbild eben jener angeblichen makrostrukturellen Unwahrheit des „Wesens überhaupt“, als welche bspw. Michael Theunissen den gesamten Zusammenhang von Reflexionsformen und Reflexionsbestimmungen unter dem Aspekt der defizienten Bestimmungslogik des Verstandes sowie der „Einheit von Darstellung und Kritik“ der Metaphysik begreift. ${ }^{125}$ Zwar erkennen sowohl der spätere Henrich als auch Iber ${ }^{126}$ in der äußeren Reflexion schließlich doch eine notwendige und erweiternde Stufe im Reflexionsbegriff: Denn erst durch das Verschwinden des Setzens in der äußeren Reflexion

123 „Dieser, (der schwerste) Theil der Logik enthält vornehmlich die Kategorien der Metaphysik und der Wissenschaften überhaupt; - als Erzeugnisse des reflectirenden Verstandes, der zugleich die Unterschiede als s el bs tä ndig annimmt, und zugleich a u ch ihre Relativität setzt; - beides aber nur neben- oder nacheinander durch ein Auch verbindet, und diese Gedanken nicht zusammenbringt, sie nicht zum Begriffe vereint.“ (GW 20, S. 145, § 114)

124 Henrich: Hegels Logik der Reflexion, S. 126. Auch Klaus J. Schmidt vertritt in seinem Kommentar zur Wesenslogik den Standpunkt, dass Hegel mit der äußeren Reflexion als Ableitung aus der setzenden Reflexion - auch aufgrund der Tatsache, dass es sich doch um einen ,relativ kleinen Abschnitt“ handle - „keine besondere Problematik verbunden zu haben“ scheint (Klaus J. Schmidt: G.W.F. Hegel. Wissenschaft der Logik - die Lehre vom Wesen: Ein einführender Kommentar, Paderborn 1997, S. 46). Darüber hinaus konstatiert auch er ihre neutrale und mehr Verwirrung stiftende Funktion: dass nämlich der „besagte Abschnitt [...] kaum als Muster einer klaren Derivation gelten [kann]. Er deutet die Problematik mehr an, als daß er sie löst“ (Schmidt: G.W.F. Hegel, S. 46). Eben dem soll hier klar widersprochen werden.

125 Theunissen: Sein und Schein, S. 14.

126 Vgl. Iber: Metaphysik absoluter Relationalität, S. 166. 
ist dieses gezwungen, sich wieder hervorzuarbeiten und sich dabei um die Aspekte der äußeren Reflexion anzureichern. In der äußeren Reflexion wird die in der setzenden Reflexion „unverarbeitete“, d.h. immer schon aufgehobene Differenz von Setzen (Reflexion) und Voraussetzen (Unmittelbarkeit) exponiert und festgehalten. Gleichwohl bilden hauptsächlich immer wieder die bloß negativen Aspekte der äußeren Reflexion, verstanden als Rückfall in ein schlechtes seinslogisches Denken, den Konsens in der Forschung: zeige doch die äußere Reflexion lediglich, dass die Verstandesmetaphysik des Wesens eigentlich nichts anderes sei als die Wiederaufnahme und Entfaltung der schlechten begrifflichen Bedingungen der Seinslogik mit ungenügenden begrifflichen Mitteln. ${ }^{127}$ Gegenstand der Kritik sind dabei, kurz zusammengefasst, die folgenden Aspekte:

(1) In der äußeren Reflexion bezieht sich die Reflexion auf das vorausgesetzte Unmittelbare als auf ein ihr Äußerliches, d. h. auf ihr eigenes Nichtsein. Äußerlich ist die Reflexion also deshalb, weil sie das Unmittelbare als „eine Voraussetzung hat “ (GW 11, S. 252), „von dem sie als von einem Fremden anzufangen“ (GW 11, S. 253) beginnt, das Reflexionsprodukt (Unmittelbarkeit) also dem Reflexionsprozess als ein Anderes zu ihm unmittelbar voraussetzt, sodass, wie Iber treffend formuliert hat, „ihr Verhältnis zu sich keines der Re-flexion mehr ist. Die äußere Reflexion ist ,negativ sich auf sich beziehende Reflexion“."128 Bezüglich der äußeren Reflexion bezeichnet Hegel mit dem Zusatz „real“ also erst einmal eine

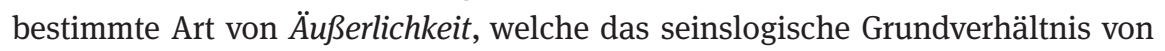
„Beziehungshaftigkeit überhaupt“ restituiert: ein Verhältnis, in welchem sich zwei Bestimmungen als äußerliche, nur andere gegeneinander gegenüberstehen - hier also das äußerliche Gegeneinander von Reflexion und Unmittelbarkeit (als Produkt der sich äußerlich werdenden Reflexion). Als „reale Reflexion“ aber ist das Negative zur Reflexion (Unmittelbare) nicht in ihr (der äußeren Reflexion) aufgehoben, sondern ihr seiendes Substrat bleibt für die Reflexion im Sinne uneinholbarer Vorgängigkeit bestehen. Daher ist der Schein der Unabhängigkeit der vorausgesetzten Unmittelbarkeit im Grunde genommen eine Abhängigkeit der Reflexion von „substratbestimmtem“ Sein als ihrer Ursache, ihremAnlass und ihrem Grund. ${ }^{129}$

(2) Eine weitere Kritik an der äußeren Reflexion bezieht sich demzufolge darauf, bloßes Verstandesdenken zu sein, das nicht spekulativ ist, weil es sich auf ein seinslogisch äußerliches, fremdes, schlechthin gegebenes Unmittelbares bezieht, durch welches es (a) absolut begrenzt wird und dem gegenüber das Denken

127 Vgl. Theunissen: Sein und Schein, S. 36.

128 Iber: Metaphysik absoluter Relationalität, S. 171.

129 Vgl. Iber: Metaphysik absoluter Relationalität, S. 172: „So ergibt sich [...] das Verhältnis der Andersheit von substratbestimmter Unmittelbarkeit und Reflexion.“ 
(b) ein nachträgliches, bloß formales Tun im Sinne der reinen und empirischen Verstandeshandlungen Kants ist. In der äußeren Reflexion bilde sich deshalb so, kurz und bündig erklärt, der alte metaphysische Realitätsbegriff ab: nämlich im Bezug der Reflexion auf die pure Positivität eines seienden, reflexionslosen Substrats, wie es sich etwa in der Realitätskategorie des Daseinskapitels zeigt. Insofern reformuliert die äußere Reflexion die verschleiernde Kategorie der Realität ${ }^{130}$, insofern in ihr gemäß der Kategorie des Daseins erneut die negativen, vermittelnden Anteile in der scheinbar ungesetzten, „pure[n] Positivität“131 des Realen verschwinden sollen. Demgemäß ist das Unmittelbare in der äußeren Reflexion ein scheinbar an sich selbst Unvermitteltes bzw. als primär unvermittelt zu Denkendes, in welchem sich die Reflexion nur nachträglich entäußern kann und welches deshalb als in seiner Vorgängigkeit schlechthin nicht vollständig Einholbares erscheint. Kants transzendentale Voraussetzung des „Ding an sich“ (vgl. Kap.1.1.5 und 1.1.6. im Hauptteil I) scheint hier erneut als der kritische Hintergrund durch, auch deshalb, weil Hegel eben diese Äußerlichkeit der Reflexion in den kantkritischen Teilen seiner Logik immer wieder herbeizitiert (vgl. auch GW 20, S. 80 f., § 44): „Der Widerspruch des Ding-an-sich besteht darin, daß es als solches außer unserer Reflexion gesetzt ist."132

(3) Der sich daraus ergebende schlechte Gegensatz von nur vorausgesetztem, bestimmungslosem Objekt und nur nachgängigem, bestimmend-erkennendem Subjekt ${ }^{133}$ führt die Reflexion aus dem Bereich der Äußerlichkeit gerade nicht in das Stadium der Innerlichkeit der setzenden Reflexion zurück. So ist eine Überwindung der äußeren Reflexion nicht durch ihre „Rückführung“, sondern einzig und allein durch ihre „Fortführung“134 möglich, die aber quasi einem Sprung zurück in den Gang des Wahren gleichkommt.

Der vorliegenden Arbeit aber soll es nicht um diese negativen Aspekte gehen, die sich besonders betonen lassen (Hegel selbst leistet dieser Betonung Vorschub, wenn er in der längeren „Anmerkung“ zum Abschnitt zur äußeren Reflexion (GW 11, S. $254 \mathrm{f}$.) diese v.a. auf die Verstandesphilosophie Kants bezieht), sondern um die für unsere Fragestellung zentralen positiven Aspekte, d.h. um die spezifischen

130 Theunissen: Sein und Schein, S. 216.

131 Iber: Metaphysik absoluter Relationalität, S. 172.

132 Iber: Metaphysik absoluter Relationalität, S. 198.

133 Iber fasst vier „wesentliche Momente des Verstandesdenkens“, welche die äußere Reflexion voraussetzt: 1. ein „unmittelbar gegebene[s] Substrat“, 2. „unmittelbar gegebene[] Bestimmungen“, 3. „Erkennen als eines bloßen Beziehens solcher unmittelbar gegebenen Bestimmungen auf ein solches Substrat“ und 4. den Gegensatz von erkennendem Subjekt und vorausgesetztem Objekt (Iber: Metaphysik absoluter Relationalität, S. 181).

134 Iber: Metaphysik absoluter Relationalität, S. 186. 
Fortschrittsaspekte der äußeren Reflexion gegenüber der setzenden Reflexion, die in der Forschung gemäß dieses Überblicks entweder verkannt oder zu einseitig und als zu ephemer betrachtet werden. Es wird sich zeigen, dass für unsere Frage nach der kategorialen Struktur von „Realität überhaupt“ gerade die äußere Reflexion von zentraler Bedeutung ist. In einem entscheidenden Abschnitt heißt es bei Hegel:

\footnotetext{
Die äusserliche Reflexion setzt also ein Seyn voraus, erstens nicht in dem Sinne, daß seine Unmittelbarkeit nur Gesetztseyn oder Moment ist, sondern vielmehr, daß diese Unmittelbarkeit die Beziehung auf sich, und die Bestimmtheit nur als Moment ist. Sie bezieht sich auf ihre Voraussetzung so, daß diese das Negative der Reflexion ist, aber so daß dieses Negative als Negatives aufgehoben ist. - Die Reflexion in ihrem Setzen, hebt unmittelbar ihr Setzen auf, so hat sie eine unmittelbare Voraussetzung. Sie findet also dasselbe vor, als ein solches, von dem sie anfängt und von dem aus sie erst das Zurückgehen in sich, das Negiren dieses ihres Negativen ist. (GW 11, S. 253)
}

Bedeutend wird die Denkfigur der Voraussetzung gerade durch den für die äußere Reflexion so zentralen „Wegfall des Setzens“ bzw. das „Gegebensein einer unmittelbaren und stabilen Voraussetzung “135, von der die Reflexion abhängt und zu der sie hinzutritt. Die äußere Reflexion ist insofern die Konsequenz der setzenden Reflexion, als das durch das Setzen vorausgesetzte Unmittelbare seine volle seinslogische Vollständigkeit wiederzuerlangen scheint und sich vom Setzungsakt der Reflexion so weit emanzipiert, dass es den Charakter eines Ungesetzten annimmt: dass es also quasi-naturalisiert hinter seine eigenen Bedingungen zurückreicht und in die Bedingungslosigkeit überzugehen scheint. So wird das Vorausgesetzte in ihr quasi isoliert fokussiert, d.h. der Aspekt des Vorausgesetztseins des Unmittelbaren tritt prägnant in den Vordergrund und der Aspekt des Gesetztseins des Unmittelbaren tritt zurück; das Unmittelbare wird nicht (mehr) als ein Produkt des Setzens der Reflexion erkannt, das es doch ebenso ist. Die äußere Reflexion scheint aus genau diesem Grund einerseits hinter die Erklärungskraft der setzenden Reflexion zurückzufallen, indem sie deren Modell vereinseitigt und verkürzt, nämlich der Aspekt des Setzens des Unmittelbaren verlorengeht. Andererseits aber - und dies wird in der Vergrößerung dieses kritischen Aspekts oft vernachlässigt - ergibt sich die äußere Reflexion erst aus der setzenden Reflexion, die sie fortführt und weshalb sie ein „Mehr an logischen Strukturen“136 gegenüber der setzenden Reflexion aufweist: Sie entsteht überhaupt erst aus der Möglichkeit der gesetzten Unmittelbarkeit und baut deren Möglichkeitsraum weiter aus. Deshalb gilt es, diesen Möglichkeitsraum mit logi-

135 Iber: Metaphysik absoluter Relationalität, S. 167.

136 Iber: Metaphysik absoluter Relationalität, S. 175. 
schen Beschreibungselementen zu füllen, und hier bietet sich im Rahmen unserer Exegese erneut die seinslogische Verantwortlichkeit an, deren grundsätzliche iterativ-regenerative Dynamik für den Anfang der Wesenslogik bereits herausgearbeitet worden ist. So verstanden, inszeniert sich die seinslogische Pendelbewegung erneut in der äußeren Reflexion: D.h. die positiven Geltungsansprüche seinslogischer Andersheit im Wesen, die Hegel seit Beginn der Wesenslogik verfolgt, erweisen sich auch in der äußeren Reflexion nur als ein scheinbarer Rückfall in schlechte, bloß defizitäre seinslogische Strukturen; folglich erweist sich auch dieser Rückgang als Entfaltung und Festigung eines berechtigten Geltungsanspruchs von integrativer Äußerlichkeit für die positiven Aspekte des Wesens, die hier als minimale kategoriale „Realität überhaupt“ gedacht werden.

Die äußere Reflexion erweist sich nun vor allem dahingehend als notwendiges und begriffserweiterndes Stadium der absoluten Reflexion, als erst hier die Reflexion anfängt, bestimmend zu werden, d.h. Bestimmtheit durch den echten, stabilen und tragenden Unterschied überhaupt erlangt. „Die Reflexion muß sich notwendig ein Sein, eine Unmittelbarkeit, voraussetzen, um sich als deren immanente Reflexion darstellen zu können. “137 Erst im völligen Durchgang der äußeren Reflexion erhält die Gesamtstruktur der Reflexion alle Bedingungen, nach denen echte reale Bestimmtheit bzw. die grundsätzliche Bestimmtheit des kategorialen minimalen „Realen überhaupt“ sinnvoll gedacht werden kann. Die „Reflexion überhaupt“ ist nämlich, das zeigt die äußere Reflexion, als ganzer Zusammenhang nur voll bestimmt und bestimmungsfähig, wenn sie von einem Unmittelbaren als Anderem zu ihr anfängt. Ihre absolute Bestimmtheit im Ganzen nämlich, d.h. die vollständige Dynamik ihres Zusammenhangs von Vermittlung als „Bestimmen überhaupt“, kann sie nicht rein durch sich selbst erzeugen, indem sie alle Bedingungen und Parameter von Setzen und Gesetzsein selbst setzt, sondern sie ist stets angewiesen auf ein Anderes als das Setzen, das sich zugleich aber nicht einfach außerhalb des logischen Raumes des Bestimmens befindet, zu welchem das Setzen gehört. Jedes „Bestimmen überhaupt“ also ist auf ein Vorgängiges, zu ihm anderes Bestimmtsein verwiesen, mit dem es denselben Raum von Bestimmtsein teilt, den es gleichwohl nicht selbst hervorbringt, um dieses überhaupt erst bestimmen zu können. Die transitive Intentionalitässtruktur von Bestimmen als logische Matrix realen Gegebenseins von etwas für jemanden ist nicht selbstgenügsam in dem Sinne, dass ihr absolut unbestimmtes Objekt als bloße Materie von reiner Bestimmungslosigkeit und reiner Bestimmungsmöglichkeit wie ein ganz Anderes zu ihm gegeben ist. „Bestimmen überhaupt“ verwirklicht sich erst dann selbstbestimmt als ein Bestimmen, wenn es auf ein An-

137 Iber: Metaphysik absoluter Relationalität, S. 169. 
deres als sein eigenes Bestimmen verwiesen ist, das aber gleichwohl an sich selbst schon bestimmt sein muss und deshalb kein Anderes gemäß der seinslogischen Ordnung von Außen/Innen, Unbestimmtheit/Bestimmtheit bzw. Unmittelbarkeit/ Vermittlung ist - in dem Sinne also, als es einen übergreifenden Raum von Bestimmtsein als logisch vorgelagerte Ordnung eines „Begrifflichen überhaupt“ konturiert, dem beide Seiten der absoluten Reflexion gerade in ihrem iterativregenerativen Anderssein zugehören: Denn auch das unmittelbare Andere in der äußeren Reflexion ist schließlich gesetzt worden, und d.h. vor allem: Es ist eine bestimmte Unmittelbarkeit im Raum des „Begrifflichen überhaupt“.

,Fortschrittlich' ist die äußere Reflexion gegenüber der setzenden Reflexion auch darin, quasi eine Metaperspektive auf das Setzen darzustellen, weil sie nämlich die Bedingungen jedes Setzens, d.h. jeden „Bestimmens überhaupt“, beschreibt: nämlich das Aussetzen des Setzens als notwendige Bedingung dafür, das Setzen in seiner Vernünftigkeit sinnvoll begreifen zu können. Die Äußerlichkeit des vorausgesetzten Unmittelbaren wird so zu einer Funktionsbeschreibung des Setzens selbst: Entscheidend ist nicht, inwiefern dieses Unmittelbare zur Sphäre des Seins dazugehört ${ }^{138}$, sondern entscheidend ist vielmehr, dass sich in ihm die Geltungsansprüche einer Andersheit artikulieren und behaupten, auf die jeder Akt des Bestimmens als auf sein intentionales Gegenstück angewiesen ist und die er nicht selbst vollständig hervorbringt (Konstruktivismus), ohne doch dabei auf ein abstrakt und gänzlich Äußerliches (im Sinne eines Verlassens des Raumes des „Begrifflichen überhaupt“) verwiesen zu sein; denn beide teilen denselben Raum von begrifflicher „Gestalthaftigkeit überhaupt“. Gerade deshalb muss auch in der äußeren Reflexion stets die Gesamtstruktur der absoluten Reflexion betrachtet werden, d.h. sowohl der Zusammenhang von Reflexion und Unmittelbarkeit als auch der Zusammenhang der drei Reflexionsformen. Denn nur so kommt entgegen der bloß kritischen Haltung gegenüber der Äußerlichkeit der Unmittelbarkeit in der äußeren Reflexion in den Blick, wie genau diese Äußerlichkeit im Kontext der iterativ-regenerativen seinslogischen Verantwortlichkeit funktioniert und woraus sie ihre unbestrittene Geltung bezieht.

Aus diesem Grund ist es wenig sinnvoll, wie Iber $^{139}$ innerhalb der äußeren Reflexion nur den Pol der vorausgesetzten Unmittelbarkeit als Statthalter des „Realen überhaupt“ im Raum des Logischen bzw. der Realität zu begreifen. Wie die vorliegende Arbeit gerade zeigen möchte, geht es in den Reflexionsformen eben nicht darum, „Realität überhaupt“ als eine fixierte Entität innerhalb über-

138 Dies richtet sich gegen Iber: Metaphysik absoluter Relationalität, S. 174, der zu sehr auf eine seinslogische Verdopplung der Reflexion im Sinne von Innen und Außen abhebt.

139 Vgl. Iber: Metaphysik absoluter Relationalität, S. 176. 
greifender logischer Strukturen zu begreifen, sondern darum, die fundamentalste logische Form von „Realität überhaupt“ selbst als eine komplexe Struktur von dynamischen Bestimmungsrichtungen zu denken, die allen isolierten und einzelnen begrifflichen Fixierungen noch vorausliegen. „Realität überhaupt“ ist also nicht bloß isolierte Positivität eines Seins, das aller Reflexion vorausliegt, sondern der komplexe Funktionszusammenhang von Bestimmen, Bestimmtsein und Bestimmtwerden sowie von Setzen, Voraussetzen und Aussetzen in dem einen großen Zusammenhang des „Begrifflichen überhaupt“, wie er sich als absolute Reflexion darstellt. Die Annahme, dass in der äußeren Reflexion die selbstbezügliche Negativität, d.h. das Setzen der absoluten Reflexion, sich im Voraussetzen als Aussetzen ihrer selbst vergisst und ein Anderes (Unmittelbares) wird $^{140}$, wodurch das Begreifen der Struktur der äußeren Reflexion doch wieder nur auf einen Begriff des vorausgesetzten Unmittelbaren als bloßen Sekundäreffekt eines sich aus den Augen verlierenden Setzens (Konstruktivismus) hinausläuft, kann daher als höchst ungenügend betrachtet werden. Stattdessen ist es sinnvoller zu durchdenken, inwiefern die absolute Reflexion bzw. das Setzen in der äußeren Reflexion seine eigene Geltung durchschaut, indem sie sich als auf ein vorausliegendes Anderes angewiesen erkennt, das aber gleichwohl Teil derselben wesenslogischen Struktur des Vermitteltseins und damit des Bestimmtseins ist.

\section{Bestimmende Reflexion}

Die bestimmende Reflexion schließlich führt diese nun bereits herausgearbeiteten Bedeutungsdimensionen der wesenslogisch vollständigen formalen Beschreibung minimaler kategorialer Realität zusammen, indem sie gemäß dem Prinzip der Aufhebung die setzende und die äußere Reflexion in der Transformation ihres gegensatzartigen Unterschieds in Momente eines Verstehenszusammenhangs vereint. Dazu heißt es:

Das Unmittelbare ist auf diese Weise nicht nur an sich, das hiesse für uns oder in der äussern Reflexion, d a s selbe, was die Reflexion ist, sondern es ist ge setzt, daß es dasselbe ist. Es ist nemlich durch die Reflexion als ihr Negatives oder als ihr Anderes bestimmt, aber sie ist es selbst, welche dieses Bestimmen negirt. - Es ist damit die Aeusserlichkeit der Reflexion gegen das Unmittelbare aufgehoben; ihr sich selbst negirendes Setzen ist das Zusammengehen ihrer mit ihrem Negativen, mit dem Unmittelbaren, und dieses Zusammengehen ist die wesentliche Unmittelbarkeit selbst. - Es ist also vorhanden, daß die

140 Iber: Metaphysik absoluter Relationalität, S. 174. 
äussere Reflexion nicht äussere, sondern eben so sehr immanente Reflexion der Unmittelbarkeit selbst ist; oder daß das was durch die setzende Reflexion ist, das an und für sich seyende Wesen ist. So ist sie bestimmende Reflexion. (GW 11, S. 253f.)

Das in der äußeren Reflexion nur gegen das Setzen der „Reflexion überhaupt“ gesetzte Vorausgesetzte des Unmittelbaren ist jetzt „ebenso sehr“ und ,in Wahrheit“ an sich selbst wieder als Setzen bestimmt, ohne damit sein Vorausgesetztsein erneut, wie in der „Setzenden Reflexion“, ganz an das Setzen zu verlieren. Folglich geht seine Andersheit und damit die seinslogische Verantwortlichkeit des Bestimmens der Reflexion gegenüber dieser Andersheit nicht erneut darin verloren, bloßer Effekt des Setzens der „Reflexion überhaupt“ zu sein: Aber die Unmittelbarkeit kann sich auch nicht mehr, wie tendenziell in der äußeren Reflexion als Rückkehr der Gefahren des „Scheins“ betont, als fundamentale Andersheit gegenüber dem Bestimmen und Reflektiertsein absoluter Negativität behaupten. Folglich führt die „bestimmende Reflexion“ erstmals ganz aus, dass auch das vorausgesetzte Unmittelbare wesentlich und tragend an einem Setzen bzw. Gesetztsein teilhat, welches jedoch nicht einfach mit dem Vollzug des Setzens der Reflexion identifiziert werden darf. Das Unmittelbare ,ist nur in der Reflexion in sich, aber es ist nicht diese Reflexion selbst“ (GW 11, S. 255): „Das Gese tz te ist daher ein Anderes, aber so, daß die Gleichheit der Reflexion mit sich schlechthin erhalten ist.“ (GW 11, S. 255)

Mit der Kennzeichnung der „Reflexion in sich“ des Unmittelbaren bezeichnet Hegel deshalb eine nun ganz wesentliche Differenzierung in der Ordnung des Raums des Begrifflichen überhaupt: Das Unmittelbare als Anderes zum Setzen der Reflexion (Setzen erster Ordnung) hat immer schon teil an einem Setzen zweiter Ordnung als einem Setzen über dem Setzen: Es ist als „Reflexion in sich“ an sich und jeder Bestimmung durch das Setzen der Reflexion Vorgängiges immer schon in sich selbst bestimmt (gesetzt). Gerade deshalb ist es für das Setzen der Reflexion unendlich bestimmbar und diesem in jeder denkbaren Weise offen. Das Setzen der Reflexion wiederum ist durch das Aussetzen, welches dem Voraussetzen der äußeren Reflexion entspringt, von dem Setzen über dem Setzen getrennt, zugleich jedoch natürlich als Vollzug derselben letztgültigen Gattung objektiven begrifflichen „Bestimmtseins überhaupt“ untrennbar mit diesem verbunden. Das Setzen über dem Setzen (Setzen zweiter Ordnung) umfasst den Unterschied von Reflexion (Setzen erster Ordnung) und Unmittelbarem, indem es ihren Widerspruch von Setzen und Voraussetzen vereint, ohne ihn auszulöschen. Daraus entsteht also ein neuer begrifflicher Hyperraum des Begrifflichen, welcher den einzelnen Akten von Setzen und Voraussetzen vorausliegen muss, damit diese überhaupt stattfinden können. 
Folglich erkennt sich die Reflexion im Unmittelbaren als ihrem Anderen als Anderes $^{141}$ : d. h. sie erkennt in ihrem vollen Unterschied zum Unmittelbaren ihren eigenen Unterschied als Setzen erster Ordnung zum Setzen zweiter Ordnung. Das aber heißt, sie erkennt sich in einer Gestalt des „Begrifflichen überhaupt“ (Setzen zweiter Ordnung), die über sie selbst als Akt setzenden Bestimmens (Setzen erster Ordnung) hinausgeht und doch nicht erneut in seinslogische Gegensätze außerhalb von „Bestimmtsein überhaupt“ zurückfällt. Wo die Reflexion im Anderen der Unmittelbarkeit zur immanenten Bewegung der Sache selbst wird (Reflexion in sich), da erkennt sich das setzende Bestimmen der Reflexion (Setzen erster Ordnung) im Unmittelbaren als an sich selbst gegen sich selbst bestimmt: so aber als Teil eines übergreifenden, dynamischen logischen Raums von objektivem Bestimmtsein (Setzen zweiter Ordnung), innerhalb dessen erst die unterschiedenen Bewegungen von Setzen, Aussetzen und Voraussetzen als einzelne stattfinden können. Man sollte deshalb keinesfalls, wie es bspw. Christian Iber tut, die bestimmende Reflexion - in Weiterführung der Kritik an der äußeren Reflexion - darauf verkürzen, hier „restituiere“ sich der „ontologisch-metaphysische Schein der Unmittelbarkeit im Wesen“142 bloß und erneut: D.h. man sollte nicht das als pure Regression verstehen, was klarerweise auch positive, realitätskategoriale Funktionalität im Sinne des argumentativen Fortschritts ermöglicht. Nur in der vollen Anerkennung der seinslogischen Veranwortlichkeit gegenüber dem Anderssein ist es möglich, eine vorausgesetzte Unmittelbarkeit als notwendigen Bezugspunkt von „Bestimmtwerden überhaupt“ (Setzen erster Ordnung) zu denken, welche gegen die Reflexion und trotzdem als in sich reflektiert, d.h. als immer schon bestimmt, widerspruchsfrei konzipiert werden kann. Deshalb ist „Realität überhaupt“ als minimales Gegebensein im Koordinatensystem von Vorliegen (Unmittelbarkeit) und Bezugnehmen (Setzen erster Ordnung) auch nicht einfach auf die Prozessualität des Setzens der Reflexion (Setzen erster Ordnung) in ihrem Unterschied von Setzen und Voraussetzen zurückzuführen ${ }^{143}$. Vielmehr differenziert sich als minimale kategoriale „Realität überhaupt“ ein übergreifender Raum von „Bestimmtsein überhaupt“ (Setzen zweiter Ordnung), gemäß der nicht-absoluten Logik seinslogischer Andersheit, in verschiedene Sphären bzw. Register des Setzens und Gesetztseins aus, ohne auf eine der in ihm eingelagerten Bestimmungsrichtungen (v.a. der des Setzens der Reflexion) zusammenzuschmelzen und deshalb entlang der starren, festen Äußerlichkeiten von Unbestimmtheit/Bestimmtheit, Unbegrifflichkeit/Begrifflichkeit, Vorliegendem/ Zugreifendem etc. letztgültig beschreibbar zu sein. Sichtbar wird hingegen vielmehr,

141 Iber: Metaphysik absoluter Relationalität, S. 191.

142 Iber: Metaphysik absoluter Relationalität, S. 193.

143 Auch dies legt, trotz anderslautender Einsichten im Laufe seiner Interpretation, Iber nahe (Iber: Metaphysik absoluter Relationalität, S. 196f.). 
dass diese Unterscheidungen nicht sinnvoll letztbegründend für einen minimalen kategorialen Begriff des „Realen überhaupt“ sein können, sondern zuallererst Sinn ergeben im Rückgang auf eine noch vorausliegende Matrix eines logisch-differenzierten Kontinuums des Begrifflichen, welches sich in zueinander andere dynamische Bezugsrichtungen von Unmittelbarkeit und Reflexion auseinanderlegt. Der kategoriale minimale Begriff von „Realität überhaupt“ zeigt sich so als Matrix von verschalteten Bedingungen von Bestimmungsrichtungen als Regelsystem darüber, wie sich feste entitätische Bestimmungen (Reflexionsbestimmungen) des Realen vernünftig minimal denken lassen müssen. Indem sich die Reflexion und ihr Setzen als ein Anderes im Unmittelbaren voraussetzen und sich als ausgesetzt erkennen, anerkennen sie einen ihnen vorausliegenden und durch sie beständig aktualisierten objektiven operativen Rahmen von „Begriffsförmigkeit überhaupt“. Dieser ist nicht auf die Aktstruktur des Setzens und ihre Konstruktionskraft zu reduzieren, sondern gibt zuallererst deren Bedingung ab, wenngleich er zum selben logischen Register von „Bestimmtheit überhaupt“ gehört. Dieser Raum gestaltet im inneren Auseinanderhalten von Unmittelbarkeit und Reflexion, von gegebenem bestimmtem Bezugspunkt alles Bestimmens und operativer Bestimmungsprozedur sowie in ihrem wechselseitigen Bedingtsein durcheinander eine komplexe Strukturformation von minimaler „Realität überhaupt“. Wie sich diese angemessene und innerhalb der Logik unwidersprochene minimale logische Struktur dann aber in ihrer Fortentwicklung derart ,überlebt‘, dass die vollständige logische Struktur des Realen nicht mehr in ihre Zuständigkeit, sondern in die Sphäre der Logik des Begriffs fällt, ist nicht mehr unser Thema.

Das Problem des Abschnitts zur „Bestimmenden Reflexion“ ist es indes, dass Hegel hier auf die Explikation der Überführung von setzender und äußerer Reflexion ineinander fast gänzlich verzichtet, diese nur mit wenigen Strichen andeutet, um die sich aus der Produktionslogik der bestimmenden Reflexion nachfolgend ergebenden Produkte von deren Tun - die Reflexionsbestimmungen - bereits in den Blick zu nehmen. Dafür stellt er die verschiedenen Arten des Gegebenseins überhaupt zwischen Seins- und Wesenslogik gegenüber, d.h. den Unterschied von seinslogischem „Dasein“ und wesenslogischem „Gesetztsein“, als Grundformen der jeweiligen festen „Bestimmtheit überhaupt“. Hegel durchdenkt dabei wiederum einen Prozess, der sich an der seinslogischen Pendelbewegung ausrichtet: indem nämlich das „Gesetztsein“ von etwas als Negation eines scheinbar Unmittelbaren (Dasein) an sich selbst, weil sich dieses eben durch die Reflexion, d.h. den Prozess begrifflicher „Vermittlung überhaupt“, bedingt findet, wiederum eine gegen diese Vermittlung durch die Reflexion gerichtete Selbstständigkeit erlangt, die es als selbstständige Bestimmung erscheinen lässt. Der „Schein“, der sich dann wiederum aus der fixierten Selbstständigkeit der Reflexionsbestimmungen gegeneinander ableitet - „als freye, im Leeren ohne 
Anziehung oder Abstossung gegen einander schwebende We sen heiten " (GW 11, S. 256) -, erweist sich als unmittelbar sich selbst aufhebend, wo „die Reflexionsbestimmtheit die Beziehung auf ihr Andersseyn an ihr selbst “ (GW 11, S. 257) darstellt: also ihre „Reflexion in sich“ in „Reflexion in Anderes“ unmittelbar übergeht und nur in dieser Einheit besteht. Damit aber sind die Überlegungen bereits zur nächsten Phase der Wesenslogik übergegangen: zu verfolgen, wie sich aus der reinen Struktur selbstbezüglicher Negativität als Keimzelle von „Bestimmtwerden überhaupt“ wieder feste, jetzt zwar wesenslogisch gebildete, aber zunehmend seinshaft fixierte Kategorien ergeben, die gemäß der „Einheit von Darstellung und Kritik“ (Theunissen) der Logik am Ende der Wesenslogik einen erneuten großen Übergang in ein Anderes, die Logik des Begriffs, notwendig machen. „Die Reflexion ist bestimmte Reflexion; somit ist das Wesen bestimmtes Wesen, oder es ist Wesen heit.“ (GW 11, S. 258) In unserer Lektüre aber ging es darum - und darauf sei am Ende der vorliegenden Untersuchung noch einmal eindringlich verwiesen -, den internen Schritt innerhalb der Wesenslogik von den Reflexionsformen zu den Reflexionsbestimmungen als einen solchen $\mathrm{zu}$ begreifen, der mit einem funktionalem Gefälle verbunden ist: Indem nämlich in der Wesenslogik zwischen dem „Ersten Kapitel“ und dem „Zweiten Kapitel“ vom Produzierenden zum Produkt, von der Reflexion zur bestimmten Reflexion, von der Dynamik wesenslogischen Bestimmens zum Bestimmtsein der Wesenheiten übergegangen wird, kommt den Überlegungen des „Ersten Kapitels“ die Möglichkeit zu, den begrifflich-genetischen Rahmen eines kategorialen minimalen Begriffs von „Realsein überhaupt“ abzugeben, welcher „,in der ,Logik der Reflexion' im Grunde methodisch thematisiert wird und [seine] fundamentale Grundstruktur zuerst in der bestimmenden Reflexion konstituiert"“144.

144 Chong-Hwa Cho: Der Begriff der Reflexion bei Hegel in Bezug auf die Wesenslogik in Hegels Wissenschaft der Logik, Berlin 2006, S. 81. 